\title{
EHG-Based Preterm Delivery Prediction Algorithm Driven by Transfer Learning
}

\author{
Yanjun $\mathrm{DENG}^{\mathrm{a}}$ and Yefei $\mathrm{ZHANG}^{\mathrm{a}}$ and Shenguan $\mathrm{WU}^{\mathrm{b}}$ and Lihuan $\mathrm{SHAO}^{\mathrm{a}, 1}$ \\ and Xiaohong ZHANG ${ }^{\mathrm{a}}$ \\ ${ }^{a}$ College of Electronics and Information Engineering, Hangzhou Dianzi University, \\ Hangzhou, China \\ ${ }^{\mathrm{b}}$ School of Communication Engineering, Hangzhou Dianzi University, Hangzhou,
} China

\begin{abstract}
Preterm delivery is currently a global concern of maternal and child health, which directly affects infants' early morbidity, and even death in several severe cases. Therefore, it is particularly important to effectively monitor the uterine contraction of perinatal pregnant women, and to make effective prediction and timely treatment for the possibility of preterm delivery. Electromyography (EHG) signal, an important measurement to predict preterm delivery in clinical practice, shows obvious consistency and correlation with the frequency and intensity of uterine contraction. This paper proposed a deep convolution neural network (DCNN) model based on transfer learning. Specifically, it is based on the VGGNet model, combined with recurrence plot (RP) analysis and transfer learning techniques such as "Fine-tune", marked as VGGNet19-I3. Optimized with the clinical measured term-preterm EHG database, it showed good auxiliary prediction performances in 78 training and test samples, and achieved a high accuracy of $97.00 \%$ in 100 validation samples.
\end{abstract}

Keywords. Electrohysterography (EHG), recurrence plot (RP), deep convolution neural network (DCNN), transfer learning, VGGNet

\section{Introduction}

Preterm birth refers to delivery more than 28 weeks but less than 37 weeks of pregnancy and is the main cause of infant morbidity and mortality. The current premature birth rate of newborns is still as high as 7\% according to the "Global Report on Premature Infants" released by the World Health Organization (WHO). Premature birth has become a global problem [1]. Therefore, it is particularly important to detect preterm birth symptoms early and carry out related treatment by implementing effective monitoring of pregnant women. The uterine contraction is an important indicator for evaluating the health of the mother and fetus during the perinatal period in clinical. As the delivery period approaches, uterine contractions gradually change from irregular and low-intensity to regular and high-intensity. Thus, it has important clinical guiding significance for the prediction of premature delivery of the fetus by operating the real-time monitoring of the uterine contractions of pregnant women.

1 Corresponding Author, Lihuan SHAO, College of Electronics and Information Engineering, Hangzhou Dianzi University, Hangzhou, China; Email: slh@hdu.edu.cn. 
Currently, the widely used clinical monitoring methods of uterine contraction mainly include internal monitoring based on intrauterine pressure catheter (IUPC) and external monitoring based on Tocodynamometer (TOCO). Both methods have certain limitations such as detection methods and accuracy. IUPC can accurately monitor uterine contractions, but its invasive is likely to cause damage to the mother and fetus. While the TOCO method can only provide information about the frequency and duration of contractions without the intensity of contractions since affected by various factors such as the placement of the probe, the thickness of subcutaneous fat, and fetal movement. And the accuracy of monitoring is also challenged.

Aiming at the shortcomings of the above detection technology, this paper proposes a fetal preterm birth prediction algorithm based on uterine Electrohysterography (EHG) signal. EHG signal is a bioelectrical signal produced by uterine activity in pregnant women. It can be collected non-invasively by a bioelectrode placed in the abdomen of the pregnant woman. The EHG signal can provide reliable information characteristics for the analysis of uterine contractions due to obvious consistency and correlation with the frequency and intensity of uterine contractions. Hence, the assisted prediction of preterm based on EHG signal has gradually become the most important method for diagnosing preterm delivery.

The main contributions of this paper include two aspects:

A fetal premature birth prediction algorithm based on EHG signal was proposed. The recursive plot (RP) analysis and transfer learning (TL) technology were integrated to construct the deep convolution neural network (DCNN) model suitable for clinical auxiliary diagnosis for realizing the intelligent auxiliary prediction of fetal premature birth.

The VGGNet model pre-trained with large sample data is migrated to the intelligent auxiliary prediction of fetal premature birth based on EHG signal using transfer learning idea. Three classical transfer learning techniques such as Fine-tune are used to optimize the model and self-adaptive learning. The serious shortage of EHG data samples is solved by realizing sufficient feature self-learning, capture and representation.

\section{Related Work}

The uterine myoelectricity of the body surface and the uterine contractions of pregnant women present a strong consistency and correlation. Therefore, the study on the prediction of fetal preterm birth based on the uterine myoelectricity has great application prospects in the clinical practice of pregnant women's health monitoring. At present, the research on premature delivery prediction of uterine electromyography is still in the early stage of development. Especially the domestic research on premature delivery prediction algorithm and system of EHG signal is still rare. Subsequently, we focus on an overview of some existing EHG research work and implementation forms under different applications.

Since the application scope of EHG signal has been widened, it is found that the accuracy of EHG signal used for uterine contraction monitoring is significantly correlated with TOCO. The in vitro detection system and the prediction algorithm of fetal premature birth based on EHG signal have been gradually studied at home and abroad. Scholars such as Oczeretko [2] and Hassan M [3] successively proposed the study of nonlinear characteristics based on EHG signal in 2005 and 2011, and found 
that EHG signal contains rich physiological information about uterine activity. Subsequently, scholars such as Fergus [4] and Ren [5] have proposed an EHG classification algorithm based on multi-angle manual feature extraction to make a preliminary auxiliary diagnosis of the risk of fetal premature birth. The accuracy of such algorithms largely depends on whether the extracted features are sufficient to accurately reflect the changing state of maternal contractions. The manual feature extraction process is relatively cumbersome, and the redundancy between different features is easy to overlook. The development of deep learning technology has made it widely used in many fields such as natural language processing and computer vision. Many scholars at home and abroad have also extended it to the research of common biomedical signals such as electrocardiogram and fetal heart rate and achieved desirable result. The fetal premature delivery prediction algorithm based on deep learning resolves the subjective of traditional machine learning and human factors through powerful feature self-learning capabilities, complex model construction and image processing technology. Since 2017, Wang Y [6], Peng J [7], Chen L [8] and other scholars have successively proposed a CNN-based Caffenet model to realize contraction recognition based on EHG signals, CNN-based fetal preterm evaluation, and deep neural network model based on sparse autoencoder to predicts the possibility of premature delivery of the fetus and achieved better performance. Although this kind of algorithm greatly simplifies the subjectivity of feature extraction, its high-precision recognition depends on abundant large sample data training. The few and insufficient EHG data of preterm in clinical application seriously restricts the training and later application of deep neural network model.

Based on the study of convolutional neural network, this paper proposes a transfer learning-based fetal premature prediction algorithm as shown in figure 1 . The nonlinear structural information characteristics in high-dimensional space of EHG signals obtained by the recurrence plot analysis are used as the input layer of the model. On the one hand, convolution neural network simplifies the subjectivity of feature extraction and reduces the project amount of the algorithm. On the other hand, the model trained on massive big data is migrated to the preterm prediction of EHG signal to solve the demand of feature self-learning for big data in deep learning algorithm.



Figure 1. Flow chart of intelligent prediction for EHG-based premature delivery via transfer learning. 


\section{EHG-Based Preterm Delivery Prediction Algorithm Driven by Transfer Learning}

\subsection{Input Layer Construction Based on Recurrence Plot Analysis}

As a non-stationary nonlinear time series, EHG contains complex physiological and pathological structure information. Based on the phase space reconstruction, the recurrence plot uses the time delay coordinates to reconstruct the phase space trajectory of the signal to reflect nonlinear characteristics in high dimensional phase space. Therefore, a recursive graph analysis tool is introduced to convert one-dimensional EHG signal into two-dimensional spatial trajectory map, which is used as the input layer data of the deep neural network model to provide sufficient high-dimensional spatial structure information for the prediction of preterm based on EHG.

(1) Phase space reconstruction to obtain phase space trajectory and space vector information.

By selecting the appropriate time delay $\tau$ and embedding dimension $\mathrm{m}$, the structure information of EHG signal in m-dimensional space is obtained, as shown in equations (1) and (2). Where the row vector $X_{k}$ in equation (1) represents the phase space vector of the $\mathrm{k}$-th point on the track, and $\mathrm{S}_{\mathrm{ij}}$ in equation (2) represents the distance between any two-phase space vector.

$$
\begin{gathered}
X=\left\{\begin{array}{c}
X_{1} \\
X_{2} \\
\vdots \\
X_{k}
\end{array}\right\}=\left\{\begin{array}{cccc}
x_{1} & x_{1+\tau} & \ldots & x_{1+(m-1) \tau} \\
x_{1} & x_{1+\tau} & \ldots & x_{1+(m-1) \tau} \\
\vdots & \vdots & \vdots & \vdots \\
x_{1} & x_{1+\tau} & \ldots & x_{1+(m-1) \tau}
\end{array}\right\} \\
\mathrm{S}_{i j}=\left\|\mathrm{X}_{i}-X_{j}\right\|, i, j \in[1, N], N=L-(m-1) \tau
\end{gathered}
$$

where L represents the length of EHG signal.

(2) Construct recurrence plot based on phase space distance.

$$
\begin{aligned}
& R_{i j}=\Theta\left(\varepsilon-S_{i j}\right) \\
& \Theta(x)=\left\{\begin{array}{l}
1, x>0 \\
0, x \leq 0
\end{array}\right.
\end{aligned}
$$

The 0-1 matrix graph, i.e., RP graph, is constructed by Heaviside function $\Theta$ of equations (3) and (4). The threshold $\varepsilon$ in equation (3) is determined by the k-nearest neighbor method $(\mathrm{k}=5)$.

The time delay $\tau$ should not be too large mainly due to the possible environmental interference and other factors. And too large $\tau$ value will introduce a large noncorrelation. In addition, the embedding dimension should also be controlled in a small range of changes, otherwise it will fall into partial structural overlap. Therefore, in the study of this paper, we select the time delay $\tau \in[1,5]$ and the embedding dimension $m \in[2,6]$ to obtain $25 \mathrm{RP}$ images of each group of EHG data. Then 
appropriately reduce the dimension and adjust each RP image element to $224 \times 224 \times 3$ to serve as the input layer of the next transfer learning algorithm. Figure 2 below shows one-dimensional waveforms and corresponding two-dimensional RP diagrams of a group of term EHG signals and a group of preterm EHG signals from the network database. Each RP map reflects the structural characteristics in the mdimensional space under time delay $\tau$ of the current fetus.


(a) EHG signal and RP map of term fetus
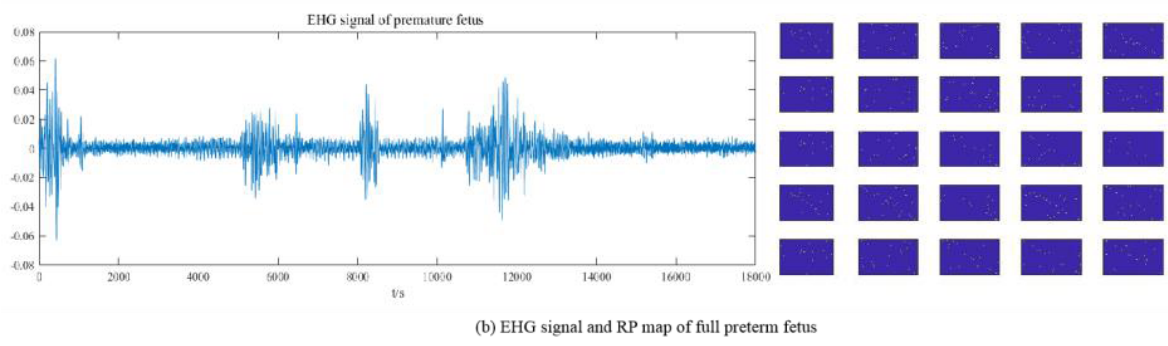

Figure 2. Recurrence plot: (a) EHG signal from term fetus, (b) EHG signal from preterm fetus.

\subsection{Deep Convolutional Neural Network Model Based on Transfer Learning}

In this paper, a deep convolution neural network model based on transfer learning is proposed to predict fetal preterm birth based on EHG signals. For the integrity of the article, this paper starts with the basic structure of CNN and the basic concepts of transfer learning, and then optimizes and adjusts the model for small sample EHG signals.

(1) Convolution neural network structure

As one of the most widely used feedforward neural networks in deep neural networks, CNN has been successfully applied in natural language processing, computer graphics, machine vision and other fields [9]. And it has become the most mature classification algorithm in pattern recognition tasks such as individual identification and medical auxiliary diagnosis.

The typical CNN model structure includes convolutional layer, excitation layer, pooling layer, fully connected layer and classifier [10], as shown in figure 3 . The convolutional layer is composed of multiple convolution kernels, which perform convolution operations with the input data to form a new feature map. The excitation layer conducts nonlinear feature mapping through classical sigmoid, tanh, ReLU and other functions. In order to reduce the number of parameters in the feature map constructed by the above two steps, the pooling layer is introduced for down-sampling operation to reduce the calculation amount in the model training process. Finally, transmit these feature information to the classifier through the full connection layer to 
achieve classification or prediction results. And the most commonly used classifiers are Softmax, support vector machine and so on. In addition, in order to avoid sample imbalance and insufficient data, the normalization layer and Dropout layer are often added to alleviate the overfitting or underfitting effect in the self-learning process of the model.

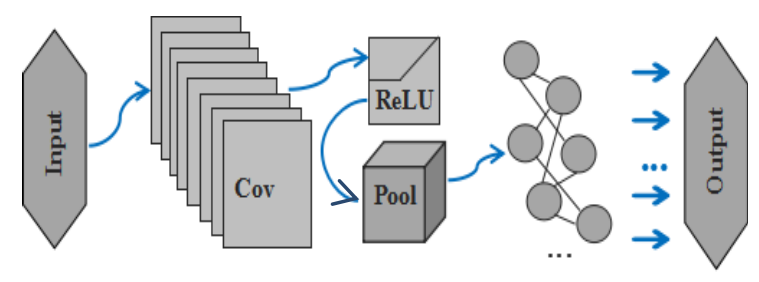

Figure 3. Basic structure of convolutional neural network.

The earliest deep CNN model is LeNet5 model proposed by LeCun in 1994, which shows strong self-learning ability in image recognition. Subsequently, classical network structures such as AlexNet, VGGNet, GoogLeNet and ResNet have been proposed and have achieved good recognition rate and equal error rate in the annual ImageNet competition [11]. One of the difficulties in the application of CNN model is the construction of network layer and the confirmation of parameters, which is a timeconsuming project and depends on repeated training and testing of a large number of samples [12]. However, it is difficult to ensure the balance of samples in clinical practice. Compared with the number of normal term fetuses, the number of preterm samples is often less. The imbalance of samples seriously restricts the construction of convolutional neural network models.

(2) Transfer learning

With the continuous development of deep learning technology, more and more researchers at home and abroad try to transfer the existing deep neural network model. Transfer learning based on model transfer is to transfer the trained model in massive large sample data to the new learning task, adjust the model structure and parameters based on the current learning task, and use the new sample data to train again to obtain the deep neural network model suitable for the current task [13].

This paper proposes a deep convolutional neural network model based on transfer learning to resolve the need for large sample data in feature self-learning when resolving small sample EHG data. Model transfer in this paper is mainly from the following two aspects:

(1) Confirm the deep neural network model to be transferred: VGGNet. The model was developed by the Computer Vision Geometry Group of Oxford University and Google DeepMind Co., Ltd., and won the runner-up of the ILSVRC competition and the champion of positioning project in 2014 . The Top5 error rate was only $7.5 \%$. The most prominent advantage of this model is its simple structure and few parameters, but excellent performance. Specifically, the model improves the recognition or classification performance by repeatedly stacking small convolution layers to form convolution groups and continuously deepening the network structure. Compared with large parameter models such as GoogLeNet and ResNet, the parameters of VGGNet are mainly concentrated in the last layers of the network and will not cause an explosion in computation. Accordingly, this article intends to build a model based on VGGNet. 
(2) Model optimization based on Fine-tune. There are three common ways to implement transfer learning: (a) Traditional transfer, which directly freezes all convolution layers of the model and only trains the full connection layer designed for the current task; (b) Transfer based on the feature vectors, which calculates and gets the final feature vector of the convolution layer of the model for the current task training and then uses the traditional machine learning method for classification and recognition; (c) Transfer based on Fine-tune, which freezes the partial convolution layer structure of the model, then learns and trains new tasks for the remaining network layers such as convolution. Compared with the first two methods, transfer model based on fine-tune not only takes advantage of the pre-training model to save time and cost, and has better robustness and generalization performance based on big data sets training, but also considers adaptability between different tasks. Based on the above two aspects, this paper proposes a deep convolutional neural network model based on VGGNet. The RP graph in 2.1 is used as the input data, and the "Fine-tune" is used to optimize the model to realize the intelligent auxiliary prediction of premature delivery based on EHG signals.

\section{Experiment and Data}

\subsection{Experimental Data}

The experimental data in this paper are from the Term-Preterm ElectroHysteroGram DataBase (TPEHGDB) at the Department of Obstetrics and Gynecology, University Medical Centre Ljubljana [14]. The database recorded the EHG signals detected by almost 1300 pregnant women in the 22nd or 32nd week of pregnancy from 1997 to 2005 with $20 \mathrm{~Hz}$ sampling frequency. Several experts such as Ivan Verdenik screened 300 groups of EHG signals from clinical and technical perspectives (including 264 groups of term delivery and 38 groups of preterm delivery data). 38 groups of preterm EHG data and 38 groups of term EHG data from 76 pregnant women were selected for experimental analysis in order to reduce the impact of sample imbalance on the model training and experimental results. And $50 \%$ of the data were used as the test set. Moreover, 100 groups of term EHG signals without model training were randomly selected from 300 groups of data as the validation set to verify the robustness of the algorithm. The sample data length of each selected group of EHG signals is 18000, which is consistent with the clinical monitoring of pregnant women's uterine contractions.

\subsection{Model Optimization and Determination}

This paper proposes three transfer models for the three commonly used transfer learning methods as shown in figure 4 and uses 38 groups of samples from TPEHG DB for model training. Then the accuracy (Acc) and F1 scores are used for performance evaluation. Figure $4 \mathrm{a}$ adopting the traditional migration method and freezing all the networks before the full connection layer is marked as VGGNet19-I1. Figure $4 \mathrm{~b}$ using the transfer learning based on feature vector and using the output of VGGNet19 model as the input of support vector machine (SVM) is marked as VGGNet19-I2. Figure 4c applying transfer model based on Fine-tune to freeze the first 13-layer network structure of the model and adjust and train the parameters of the latter 
6-layer structure is marked as VGGNet19-I3. The training parameters of the model are set as follows: the initial learning rate is $0.2 \times 10^{-3}$ and the number of iterations of the model is 500. The experimental result is obtained as shown in table 1 which based on model training and testing of 76 sets of data and validating of 100 sets of untrained term data. The performance of VGGNet19-I1 and VGGNet19-I2 is highly consistent in training set, test set and validation set, which reflects the strong feature self-learning and adaptive ability of the original VGGNet to a certain extent and can achieve more accurate classification tasks only with simple classifiers. The performance of VGGNet19-I3 is significantly better than the first two. The reason is mainly due to the parameter optimization advantage of Fine-tune. For any deep neural network model, the first several layers mainly learn the general characteristics of the current task and the latter several layers mainly learn to adapt to the special characteristics of the current task. Therefore, it is necessary to carry out certain training and adaptive learning for the current task even if it is a pre-training model. In this paper, VGGNet19-I3 network model is finally selected as the model of the auxiliary prediction algorithm of fetal premature birth based on EHG.

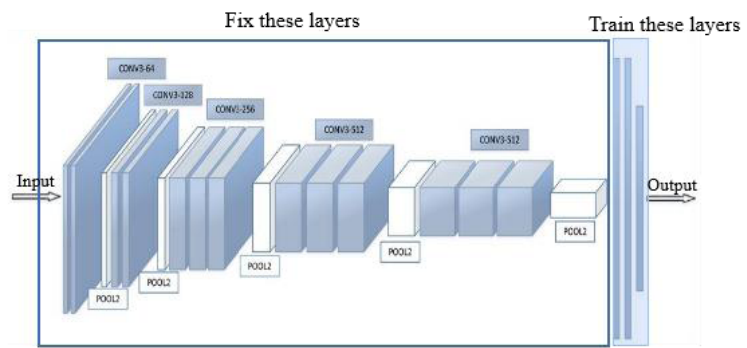

(a)

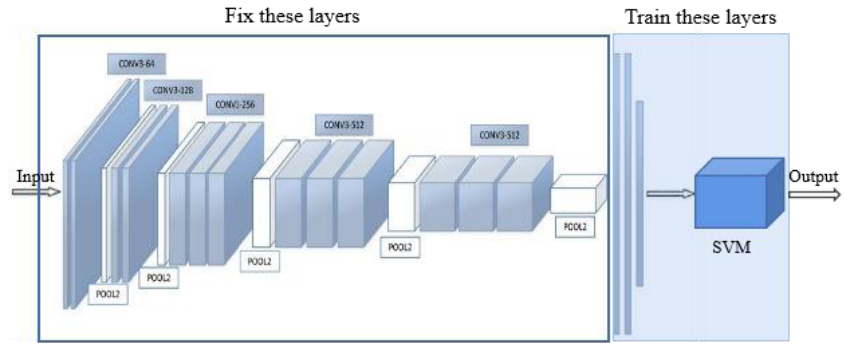

(b)

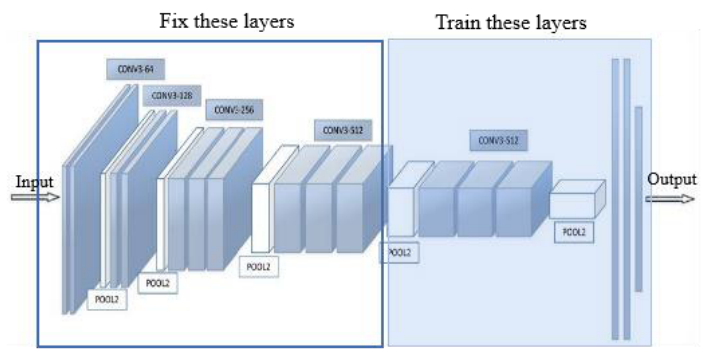

(c)

Figure 4. Model optimization based on VGGNet: (a) traditional TL, (b) feature vector-based TL, (c) Finetune-based TL. 
Table 1. Experimental results of VGGNet19-In.

\begin{tabular}{lllllll}
\hline & \multicolumn{2}{c}{ Training set } & \multicolumn{2}{c}{ Test set } & \multicolumn{2}{c}{ Validation set } \\
\cline { 2 - 7 } & Acc & F1 & Acc & F1 & Acc & F1 \\
\hline VGGNet19-I1 & 90.84 & 90.72 & 81.63 & 81.33 & 80.50 & 80.79 \\
VGGNet19-I2 & 90.73 & 90.61 & 82.79 & 82.52 & 82.00 & 82.52 \\
VGGNet19-I3 & 92.95 & 93.01 & 92.11 & 91.98 & $\mathbf{9 7 . 0 0}$ & $\mathbf{9 6 . 9 7}$ \\
\hline
\end{tabular}

\subsection{Experimental Results and Analysis}

In order to make a more objective analysis of the proposed VGGNet19-I3 model, the typical domestic and foreign studies in recent years are listed in table 2 below.

Table 2. Comparison of different algorithms.

\begin{tabular}{lll}
\hline Author & Classifier & Performance \\
\hline John N.S, 2020 [15] & KNN & Acc $=90.32 \%$ \\
Khan M U, 2019 [16] & SVM & Acc $=95.5 \%$ \\
Chen L L, 2020 [8] & SSAE+DNN & Acc $=97.9 \%$ \\
Proposed & RP+VGGNet19-I3 & Acc $=97.00 \%$ \\
\hline
\end{tabular}

The comparison shows that the proposed algorithm has better classification performance. Although the algorithm proposed by Chen L L [8] achieves $97.9 \%$ accuracy, it is more complex than the algorithm proposed in this paper.

\section{Conclusion}

Perinatal uterine contraction monitoring of pregnant women has important clinical application value for effective prediction and timely treatment of premature delivery. This paper constructs a fetal premature prediction algorithm of EHG using VGGNet19I3 based on transfer learning technology and proposes a feasible and practical method for clinical auxiliary diagnosis of fetal premature. $\mathrm{CNN}$ and other deep neural network algorithms greatly simplify the subjectivity of feature extraction. But their high accuracy still relies on abundant large samples, which is not suitable for EHG signal analysis with insufficient data samples. Therefore, this paper adopts the transfer learning technology to migrate the VGGNet model pre-trained on large sample data. In order to make the model better applicable to the classification task of EHG signals, this paper adopts three ways of traditional migration, feature vector-based migration and Fine-tune-based migration to optimize and adaptively learn the model to realize the intelligent auxiliary prediction of fetal premature birth of EHG signals with small samples. In future research, we will focus on the optimal design of EHG signals to achieve better data acquisition.

\section{Acknowledgement}

This work was supported in part by Basic Public Welfare Research Project in Zhejiang Province (LGG19F010010). 


\section{References}

[1] Karlsson B, Terrien J, et al. Abdominal ERG on a 4 by 4 grid: mapping and presenting the propagation of uterine contractions[C]. Mediterranean Conference on Medical \& Biomedical Engineering \& Computing: 2007: 139-143.

[2] Oczeretko E, Kitlas A, et al. Nonlinear dynamics in uterine contractions analysis[J]. Math Biosci Interac. 2005:215-222.

[3] Hassan M, Boudaoud S, et al. Combination of Canonical Correlation Analysis and Empirical Mode Decomposition Applied to Denoising the Labor Electrohysterogram[J]. IEEE Transactions on Biomedical Engineering: 2011;58(9):2441-2447.

[4] Fergus P, Cheung P, et al. Prediction of preterm deliveries from EHG signals using machine learning[J]. PLoS One: 2013 8(10): e77154.

[5] Ren P, Yao S, et al. Improved prediction of preterm delivery using empirical mode decomposition analysis of uterine electromyography signals[J]. PLoS One: 201510 (7): e0132116.

[6] WANG Y. Study on uterine contraction recognition based on convolution neural network [D], Beijing, 2019

[7] PENG J, HAO D M, et al. Preliminary Study on the Efficient Electrohysterogram Segments for Recognizing Uterine Contractions with Convolutional Neural Networks[J]. Biomed Research International. 2019: 3168541-3168541.

[8] CHEN L L, XU H Y. Deep neural network for semi-automatic classification of term and preterm uterine recordings [J]. Artificial Intelligence in Medicine: 2020, 105.

[9] Krizhevsky A, Sutskever I, Hinton G. ImageNet Classification with Deep Convolutional Neural Networks[J]. Advances in neural information processing systems, 2012, 25(2).

[10] LAN J H, WANG D, SHEN X P. Research progress on visual image detection based on convolutional neural network [J]. Chinese Journal of Scientific Instrument, 2020,41(04):167-182.

[11] WANG Y Z, ZHAO W, XU H Y, LIU J Y. Non-fixed scene weather classification algorithm based on lightweight convolutional neural network[J]. Electronic Measurement Technology, 2019, 42(17): 152156.

[12] WU R X, XIAO Q K. Multi-object images recognition based on deep networks and data augmentation [J]. Foreign Electronic Measurement Technology, 2019, 38(05): 86-90.

[13] LI W T, HAN H H, JIAO D, TANG J, DING M S. Research on intelligent cognition method of foglevel based on deep transfer learning[J]. Journal of Electronic Measurement and Instrumentation, 2020, 34(02): 88-96.

[14] Gašper Fele-Žorž, Gorazd Kavšek, Živa Novak-Antolič and Franc Jager. A comparison of various linear and non-linear signal processing techniques to separate uterine EMG records of term and preterm delivery groups. Medical \& Biological Engineering \& Computing, 46(9):911-922 (2008).

[15] John N.S, Sriraam N, Martis R.J, et al. Computational Method for Preterm Labor Prediction using Electrohysterogram[C]. //2020 IEEE International Conference on Distributed Computing, VLSI, Electrical Circuits and Robotics (DISCOVER). IEEE, 2020.

[16] Khan M U, Aziz S, Ibraheem S, et al. Characterization of Term and Preterm Deliveries using Electrohysterograms Signatures[C]. //2019 IEEE 10th Annual Information Technology, Electronics and Mobile Communication Conference (IEMCON). IEEE, 2019. 